\section{Terapia de inducción acelerada con infliximab en paciente con colitis ulcerosa grave refractaria a corticoides. Caso clínico}

\author{
DANIELA FLUXÁa ${ }^{\mathrm{a}}$, LILIAN FLORES ${ }^{1, \mathrm{~b}}$, UDO KRONBERG $^{1,2}$, \\ MAURICIO MORENO ${ }^{3, c}$, CAROLINA FIGUEROA $^{1,4}$, \\ PATRICIO IBÁÑEZ ${ }^{1,4}$, JAIME LUBASCHER ${ }^{1,4}$, \\ DANIELA SIMIAN ${ }^{1,5, \mathrm{~b}}$, RODRIGO QUERA $^{1,4}$
}

\section{Acute severe ulcerative colitis treated with accelerated infliximab induction. Case report}

Acute severe ulcerative colitis (ASUC) is a potentially life-threatening condition that requires early recognition, hospitalization and adequate treatment. Currently, the use of infliximab in ulcerative colitis (UC) is recommended in the case of severe disease refractory to corticosteroids, once that superimposed bacterial or viral infections (such as cytomegalovirus or Clostridium difficile) have been excluded. However, conventional weight-based regimens of infliximab might be insufficient for patients with ASUC. Accelerated infliximab induction regimen may increase its serum concentration levels and efficacy by reducing early colectomy rates in these patients. We report a 34 year old female presenting with an ASUC. She was initially treated with hydrocortisone $300 \mathrm{mg} /$ day and mesalazine enemas $4 \mathrm{~g} /$ day with an unfavorable clinical response. At the fifth day of therapy, an accelerated induction therapy with infliximab was started in doses of $10 \mathrm{mg} / \mathrm{kg}$ at weeks 0,1 and 4 . After the second dose, there was a favorable response with reduction of abdominal pain, stool frequency and hematochezia. She was discharged with prednisone and azathioprine. After a year of starting infliximab, the patient remains in clinical remission.

(Rev Med Chile 2017; 145: 1083-1088)

Key words: Colitis, Ulcerative; Infliximab; time-To-treatment.

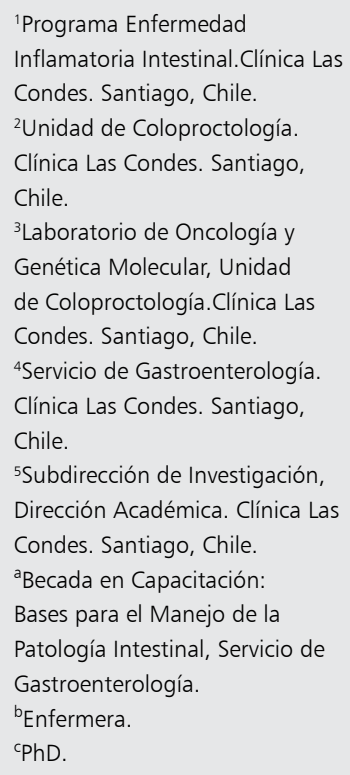

Conflicto de intereses: No existen conflictos de intereses. Fuente de apoyo financiero: Proyecto Clínica Las Condes N ${ }^{\circ}$ PIDA2015-003.

Recibido el de 20 enero de 2017, aceptado el 28 de agosto de 2017.

Correspondencia a:

Dr. Rodrigo Quera

Clínica Las Condes Estoril 450, Las Condes, Santiago, Chile. rquera@clc.cl
L

a colitis ulcerosa grave (CUg) constituye una emergencia médica. Aunque los corticoesteroides intravenosos han sido la principal estrategia, un tercio de los pacientes no responden a esta terapia ${ }^{1}$. Infliximab, ciclosporina y tacrolimus han sido utilizados como terapia de rescate disminuyendo la necesidad de colectomía precoz $^{2-6}$

El actual esquema de inducción de infliximab en CUg está basado en los estudios ACT, los que excluyen a este grupo de pacientes ${ }^{7}$. Además, se ha demostrado que el riesgo de colectomía precoz en CUg es mayor que en casos más leves ${ }^{8}$.

$\mathrm{La}$ CUg se asocia con un mayor nivel circulante de factor de necrosis tumoral (TNF- $\alpha)^{9}$, que se traduce en una mayor actividad inflamatoria, un mayor clearance del fármaco ${ }^{10}$ y una pérdida de éste en las deposiciones ${ }^{11}$. Este escenario sugiere que estos pacientes podrían requerir un esquema de inducción con intervalos menores y/o una 
mayor dosis de infliximab para mantener niveles terapéuticos adecuados ${ }^{12}$. Gibson y cols. han demostrado que un esquema de inducción acelerada (EIA) en pacientes con CUg disminuye la necesidad de colectomía precoz ${ }^{13}$. Presentamos un caso clínico en el cual esta estrategia fue factible, efectiva y segura.

\section{Caso clínico}

Mujer de 34 años sin antecedentes de importancia, quien presenta en junio de 2016 cuadro de tres semanas de evolución caracterizado por deposiciones líquidas (frecuencia ocho veces/ día y tres/noche) con sangre fresca y coágulos. Posteriormente se agrega dolor cólico en hemiabdomen izquierdo y fiebre hasta $37,8^{\circ} \mathrm{C}$ motivo por el cual es hospitalizada. Se realizan exámenes de laboratorio donde destacan Hcto/Hb 27\%/9,3 g/ dl; leucocitos 12.300 , proteína $\mathrm{C}$ reactiva (PCR) $117 \mathrm{mg} / \mathrm{L}$ (normal < 10), potasio $2,83 \mathrm{mEq} / \mathrm{l}$ y albúmina $2,7 \mathrm{~g} / \mathrm{dl}$. PCR para Clostridium difficile negativo y calprotectina fecal $(\mathrm{CF})>3.000 \mathrm{ug} / \mathrm{g}$. La tomografía computada de abdomen-pelvis muestra un engrosamiento difuso del colon y recto. La colonoscopía sin ileoscopía confirma compromiso difuso desde colon transverso proximal a recto con presencia de úlceras profundas (Ulcerative Colitis Endoscopic Severity Index [UCEIS-8] ${ }^{14}$ (Figura 1 A-D). Las biopsias muestran un compromiso inflamatorio crónico (alteración de la arquitectura) y agudo (criptitis y abscesos crípticos) con un índice histológico de Geboes de 5,3 ${ }^{15}$. Estudio de citomegalovirus por inmunotinción y reacción polimerasa en cadena en biopsias negativos. Se inicia tratamiento con corticoesteroides (hidrocortisona intravenosa $300 \mathrm{mg} /$ día) y enemas de mesalazina $4 \mathrm{~g} /$ noche. La respuesta clínica es desfavorable persistiendo con deposiciones líquidas con sangre (seis veces/ día) y dolor abdominal, motivo por el cual se decide al quinto día iniciar infliximab con $\operatorname{EIA~(0,~}$ 1 y 4 semanas) en dosis de $10 \mathrm{mg} / \mathrm{kg}$. Tras la $2 \mathrm{da}$ dosis, la paciente evoluciona en forma favorable con disminución progresiva del dolor abdominal, deposiciones formadas dos veces/día con trazas de sangre siendo dada de alta con prednisona $40 \mathrm{mg} /$ día con disminución rápida y azatioprina $175 \mathrm{mg} /$ día. A las 4 semanas del inicio de infliximab se mantiene en remisión clínica (índice de Mayo parcial-0) con CF $426 \mathrm{ug} / \mathrm{g}$. Previo a la $4 \mathrm{a}$ dosis (semana 12, dosis $5 \mathrm{mg} / \mathrm{kg}$ ) se realizan exámenes de control destacando Hcto/Hb 42\%/13,9 g/dl; leucocitos 6.170, plaquetas 379.000, PCR $3 \mathrm{mg} / \mathrm{L}$, albúmina 4,3 gr/dl, CF 169 ug/g y colonoscopía que muestra remisión endoscópica (curación de la mucosa) con un UCEIS-0, describiéndose sólo la presencia de pólipos inflamatorios en colon descendente y sigmoides (Figura $1 \mathrm{E}-\mathrm{H}$ ). Las biopsias muestran ausencia de alteraciones en la arquitectura y presencia aislada de un pequeño
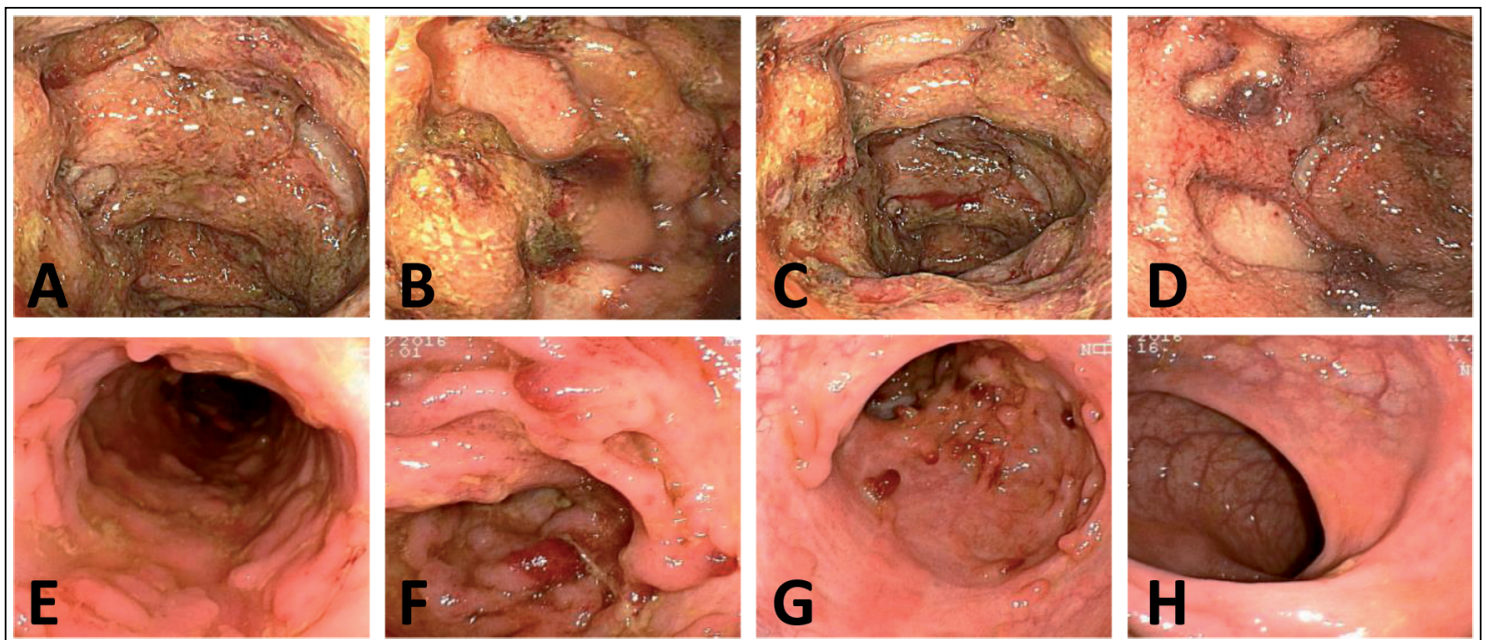

Figura 1. Colonoscopía previo al inicio de Infliximab (A-D) compatible con Ulcerative Colitis Endoscopic Severity Index (UCEIS-8) y previo a la $4^{\text {a }}$ dosis de este biológico (E-H) mostrando un UCEIS-0. 
foco de criptitis (índice de Geboes 3,1). Los niveles de infliximab y anticuerpos contra infliximab tomados previo a la administración de la 4a dosis fueron de $1,1 \mathrm{ug} / \mathrm{mL}$ y $5,8 \mathrm{UA} / \mathrm{mL}$, respectivamente. A la semana 52 (9a dosis de infliximab), la paciente se mantiene en remisión clínica (índice parcial de Mayo-0) con Hcto/Hb 40\%/13,6 g/dl; leucocitos 9.240, plaquetas 332.000, PCR $1 \mathrm{mg} / \mathrm{L}$ y CF $45 \mathrm{ug} / \mathrm{g}$.

\section{Discusión}

Aunque infliximab, adalimumab, golimumab y vedolizumab han sido aprobados por la Food and Drug Administration (FDA) y la European Medicine Agency (EMA) ${ }^{16}$, sólo infliximab ha sido sugerido en pacientes con $\mathrm{CUg}^{2-6}$. Por ello, es importante considerar la presencia de factores que pueden modificar la farmacocinética de infliximab y con ello los niveles plasmáticos y tisulares del fármaco ${ }^{17-19}$. Niveles elevados de TNF- $\alpha$ a nivel plasmático y tisular, PCR elevada, hipoalbuminemia y un índice de masa corporal elevado, pueden aumentar el clearence de infliximab afectando de esta manera su efectividad. Por otra parte, la pérdida del fármaco en las deposiciones ha sido asociada a una menor respuesta primaria en pacientes con $\mathrm{CUg}^{11}$.

En nuestra paciente destacaba al ingreso la presencia de PCR $117 \mathrm{mg} / \mathrm{L}$, albúmina 2,7 g/dl y colonoscopía con compromiso extenso con presencia de úlceras profundas en la mucosa (UCEIS-8).

Parece racional sugerir, por lo previamente expuesto, que frente a una CUg sea necesario modificar el esquema de inducción ya sea acortando los intervalos de las dosis ( 0,1 y 4 semanas) y/o aumentando la dosis del biológico $(10 \mathrm{mg} / \mathrm{kg})^{12}$. A pesar de que en las guías de manejo de Colitis Ulcerosa (CU) no está establecido el uso de un EIA en el tratamiento de la CUg, un estudio mostró que el $76 \%$ de los encuestados utilizaba esta estrategia en pacientes hospitalizados por $\mathrm{CUg}^{20}$. En la actualidad, sólo tres estudios han planteado su uso durante la fase de inducción en pacientes con $\mathrm{CUg}^{13,21,22}$. En el único estudio publicado en extenso, Gibson y cols. han sigerido que un EIA podría disminuir el riesgo de colectomía precoz sin afectar la necesidad de cirugía a mediano o largo plazo $^{13}$.

Nuestra paciente ya ha completado un año desde su crisis grave refractaria a corticoesteroides logrando el objetivo de evitar una colectomía precoz. Aunque la paciente requiriera una cirugía en el futuro, se habrá logrado cambiar una indicación de emergencia por una electiva, disminuyendo así la morbilidad asociada.

La decisión de acortar el intervalo de administración versus aumentar la dosis puede estar fundamentada en la farmacocinética de infliximab. Aunque mayores dosis sean utilizadas, un clearance aumentado del fármaco afectará los niveles hacia el final de cada intervalo ${ }^{23,24}$. En nuestra paciente decidimos utilizar un esquema acelerado (0, 1 y 4 semanas) con aumento de la dosis administrada $(10 \mathrm{mg} / \mathrm{kg})$ dado la severidad clínica y endoscópica ${ }^{12}$.

En esta paciente, el EIA fue efectivo, no sólo al alcanzar la remisión clínica (índice parcial de Mayo-0), sino también al lograr normalizar valores de PCR, disminuir los niveles de $\mathrm{CF}$ a rangos aceptables y finalmente un control endoscópico previo a la 4 a dosis de infliximab que demuestra una curación de la mucosa (UCEIS-0). La disminución de los valores de PCR hasta lograr valores normales ha sido planteada dentro del algoritmo de manejo de pacientes con Enfermedad Inflamatoria Intestinal (EII), asociándose a una respuesta clínica sostenida ${ }^{25}$. En relación a CF, estudios han demostrado que pacientes con $\mathrm{CUg}$ con $\mathrm{CF}>1.922 \mathrm{ug} / \mathrm{g}$ presentan un mayor riesgo de colectomía, situación que se dio en nuestra paciente ${ }^{26}$. La evolución de este biomarcador ha sido favorable logrando alcanzar a la semana 52 un valor de $45 \mathrm{ug} / \mathrm{g}$ (Figura 2). Los dos últimos resultados (semana 28 y 52) se encuentran dentro del rango sugerido en los algoritmos de seguimiento en pacientes con $\mathrm{CU}^{27}$.

La medición de niveles de infliximab y anticuerpos contra este y otros biológicos ha permitido optimizar la terapia en pacientes con EII. Sin embargo, debe considerarse que el papel de esta estrategia está dado por los distintos escenarios clínicos que presenten los pacientes al momento de la medición ${ }^{28}$. En CU activa, niveles elevados de infliximab presentarían un mayor porcentaje de remisión y menor frecuencia de colectomía al compararlos con aquellos que presentan niveles indetectables ${ }^{29}$. Sin embargo, Ungar y cols. demostraron recientemente que en pacientes con CUg, el grupo con falta de respuesta primaria no presentó niveles de infliximab menores que 


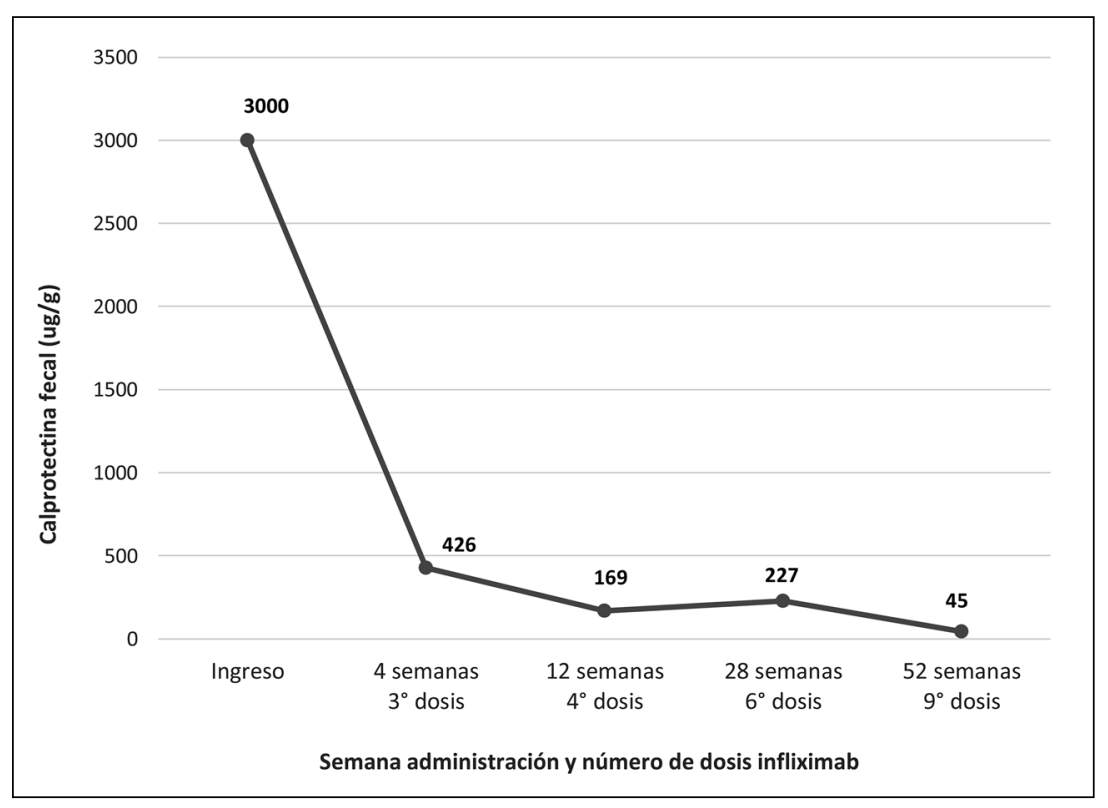

Figura 2. Evolución de calprotectina fecal durante el tratamiento con infliximab en esquema de inducción acelerada (dosis $10 \mathrm{mg} / \mathrm{Kg}$ ). el grupo que respondió a dosis estándar de infliximab $^{30}$. Niveles de infliximab/TNF- $\alpha$ tisular o la pérdida de este fármaco por las deposiciones, deben ser considerados al evaluar la efectividad de esta terapia ${ }^{19}$. En nuestra paciente los niveles de infliximab y sus anticuerpos fueron medidos como parte de un protocolo de investigación antes de la 4a dosis (semana 12). Los niveles bajos de infliximab $(<3,5 \mathrm{ug} / \mathrm{mL})^{28}$, pese a utilizar una dosis de $10 \mathrm{mg} / \mathrm{kg}$ en las tres dosis de inducción, sugieren un mayor clearance de este fármaco. Estos niveles bajos no se explicarían por una reacción inmune contra el biológico dado que los niveles de los anticuerpos contra este son clínicamente irrelevantes $(<7 \mathrm{UA} / \mathrm{mL})^{17,28}$.

En conclusión, de acuerdo a lo planteado por Gibson y cols. ${ }^{13}$ y según el resultado de nuestra paciente, un EIA con infliximab podría ser considerado en pacientes con CUg refractaria a corticoesteroides con el fin de disminuir el riesgo de colectomía precoz. Dado que recientemente se ha reportado un evento adverso fatal post esquema de inducción acelerada con infliximab ${ }^{31}$, creemos que es fundamental utilizar todos los criterios de gravedad antes de decidir el uso de esta estrategia, la cual debe estar bajo un esquema protocolizado. Estudios prospectivos y aleatorizados permitirán definir el rol de la medición de niveles de inflixi- mab y anticuerpos contra este biológico en este escenario.

\section{Referencias}

1. Turner D, Walsh CM, Steinhart AH, Griffiths AM. Response to corticosteroids in severe ulcerative colitis: a systematic review of the literature and a meta-regression. Clin Gastroenterol Hepatol 2007; 5: 103-10.

2. Narula N, Marshall JK, Colombel JF, Leontiadis GI, Williams JG, Muqtadir Z, et al. Systematic review and meta-analysis: Infliximab or cyclosporine as rescue therapy in patients with severe ulcerative colitis refractory to steroids. Am J Gastroenterol 2016; 11: 477-91.

3. Feuerstein JD, Akbari M, Tapper EB, Cheifetz AS. Systematic review and meta-analysis of third-line salvage therapy with infliximab or cyclosporine in severe ulcerative colitis. Ann Gastroenterol 2016; 29: 341-7.

4. Komaki Y, Komaki F, Micic D, Yamada A, Suzuki Y and Skuraba A. Pharmacological therapies for severe steroid refractory hospitalized ulcerative colitis: a network meta-analysis. J Gastroenterol Hepatol 2016 Dec 13.

5. Seah D, De Cruz P. Review article: the practical management of acute severe ulcerative colitis. Aliment Pharmacol Ther 2016; 43: 482-513.

6. Hernández-Rocha C, Ibáñez P, Molina ME, Klassen J, Valenzuela A, Candia R, et al. Diagnóstico y manejo 
de Colitis Ulcerosa grave. Una mirada actualizada. Rev Med Chile 2017; 145: 96-105.

7. Rutgeerts P, Sandborn WJ, Feagan BG, Reinisch W, Olson A, Johanns J, et al. Infliximab for induction and maintenance therapy for ulcerative colitis. N Engl J Med 2005; 353: 2462-76.

8. Sjöberg M, Magnuson A, Bjork J, Benoni C, Almer S, Friis-Liby I, et al. Infliximab as rescue therapy in hospitalized patients with steroid-refractory acute ulcerative colitis: a long-term follow-up of 211 Swedish patients. Aliment Pharmacol Ther 2013; 38: 377-87.

9. Gardiner KR, Halliday MI, Barclay GR, Milne L, Brown D, Stephens S, et al. Significance of systemic endotoxaemia in inflammatory Bowel disease. Gut 1995; 36: 897901.

10. Kevans D, Murthy S, Iacono A, Silverberg MS and Greenberg GR. Accelerated clearance of infliximab during induction therapy for acute ulcerative colitis is associated with treatment failure. Gastroenterology 2012; 142: S-384-5.

11. Brandse JF, van der Brink GR, Wildenberg ME, van der Kleij D, Rispens T, Jansen JM, et al. Loss of infliximab into feces is associated with lack of response to therapy in patients with severe ulcerative colitis. Gastroenterology 2015; 149: 350-5.

12. Hindryckx P, Novak G, Vande Casteele N, Laukens D, Parker C, Shackelton LM, et al. Review article: dose optimisation of infliximab for acute severe ulcerative colitis. Aliment Pharmacol Ther 2017; 45: 617-30.

13. Gibson DJ, Heetun ZS, Redmond CE, Nanda KS, Keegan D, Byrne K, et al. An accelerated infliximab induction regimen reduces the need for early colectomy in patients with acute severe ulcerative colitis. Clin Gastroenterol Hepatol 2015; 13: 330-5.

14. Walsh A, Palmer R, Travis S. Mucosal healing as a target of therapy for colonic inflammatory bowel disease and methods to score disease activity. Gastrontest Endosc Clin N Am 2014; 24: 367-78.

15. Geboes K, Kiddell R, Öst A, Jensfelt B, Persson T, Löfberg R. A reproducible grading scale for histological assessment of inflammation in ulcerative colitis. Gut 2000; 47: 404-9.

16. Macaluso FS, Renna S, Orlando A, Cottone M. The biologics of ulcerative colitis. Expert Opinion Biol Ther 2017; 17: 175-84.

17. Ordás I, Mould DR, Feagan BR and Sandborn WJ. Anti-TNF monoclonal antibodies in inflammatory bowel disease: pharmacokinetics-based closing paradigms. Clin Pharmacol Ther 2012; 91: 635-46.

18. Rosen MJ, Minar P, Vinks AA. Review article: applying pharmacokinetics to optimize dosing of anti-TNF biolo- gics in acute severe ulcerative colitis. Aliment Pharmacol Ther 2015; 41: 1094-103.

19. Yarur AJ, Jain A, Sussman DA, Barkin JM, Quintero MA, Princen F, et al. The association of tissue anti-TNF drug levels with serological and endoscopic disease activity in inflammatory bowel disease: the ATLAS study. Gut 2016; 65: 249-55.

20. Herfarth HH, Rogler G, Higgins PD, Pushing the pedal to metal: should we accelerate infliximab therapy for patients with severe ulcerative colitis?. Clin Gastroenterol Hepatol 2015; 13: 336-68.

21. Govani SM, Waljee AK, Stidham RW, Higgins P, Hardiman K, Accelerated dosing of infliximab prevents colectomy within day in only half of patients with severe ulcerative colitis. Gastroenterology 2016; 1: S106.

22. Choy MC, Seah D, Gorelik A, Macrae FA, Sparrow M, Connell W, et al. Comparison of accelerated infliximab induction vs standard induction treatment in acute severe ulcerative colitis. Gastroenterology 2016; 1: S803.

23. Dotan I, Ron Y, Yanai H, Becker S, Fishman S, Yahav L, et al. Patients factors that increase infliximab clearance and shorther half-life in inflammatory bowel disease: A population pharmacokinetic study. Inflamm Bowel Dis 2014; 20: 2247-59.

24. Katz L, Gisbert JP, Manoogian B, Lin K, Steenholdt C, Mantzaris GJ, et al. Doubling the infliximab dose versus halving the intervals in Crohn's disease patients with loss of response. Inflamm Bowel Disease 2012; 18: 2026-33.

25. Reinisch W, Wang Y, Oddens BJ and Link R. C-reactive protein, an indicator for maintained response of remission to infliximab in patients with Crohn's disease: a post-hoc analysis from ACCENT I. Aliment Pharmacol Ther 2012; 35: 568-76.

26. Ho GT, Lee HM, Brydon G, Ting T, Hare N, Drummond $\mathrm{H}$, et al. Fecal calprotectin predicts the clinical course of acute ulcerative colitis. Am J Gastroenterol 2009; 104: 673-8.

27. Lin JF, Chen JM, Zuo JK, Yu A, Xiao ZJ, Deng FH, et al. Meta-analysis fecal calprotectin for assessment of Inflammatory Bowel Disease activity. Inflamm Bowel Dis 2014; 20: 1407-15.

28. Melmed GY, Irving PM, Jones J, Kaplan GG, Kozuch PL, Velayos FS. Appropriateness of testing for anti-tumor necrosis factor agent and antibody concentrations, and interpretation results. Clin Gastroenterol Hepatol 2016; 14: $1302-9$.

29. Seow CH, Newman A, Irwin SP, Steinhart AH, Silverberg MS, Greenberg GR. Trough serum infliximab: a predictive factor of clinical outcome for infliximab treatment in acute ulcerative colitis. Gut 2010; 59: 49-54. 
30. Ungar B, Mazor Y, Weisshof R, Yanai H, Ron Y, et al. Induction infliximab levels among patients with acute severe ulcerative colitis compared with patients with moderately severe ulcerative colitis. Aliment Pharmacol Ther 2016; 43: 1293-9.
31. Rofaiel R, Kohli S, Mura M, Hosseini-Moghaddam SM. A 53-year-old man with dyspnea, respiratory failure, consistent with infliximab-induced acute interstitial pneumonitis after an accelerated induction dosing schedule. BMJ Case Rep 2017 May 12: 2017. 